\title{
CANDIDA ALBICANS “FUNGUS BALL” IN BLADDER
}

\author{
DAVID L. HAROLD, M.D. \\ STEPHEN A. KOFF, M.D. \\ EVAN J. KASS, M.D.
}

From the Sections of Urology, Departments of Surgery, University of Michigan Medical Center and Ann Arbor

Veterans Administration Hospital, Ann Arbor, Michigan

ABSTRACT - With the use of antibacterial agents, the incidence of fungal infections has risen. The urinary tract is susceptible to these superinfections. We report, herein, a case of candidiasis of the bladder that demonstrates the classic radiographic findings of "fungus ball" in the bladder.

A sixty-four-year-old white male presented in acute urinary retention with urosepsis, congestive heart failure, and severely uncontrolled diabetes mellitus. One year prior to admission he was hospitalized and required Foley catheter drainage and antibiotics. At that time he was diagnosed as having a ruptured prostatic abscess, and purulent material drained per urethra for several days after catheter removal.

The patient voided freely until four months prior to this admission when obstructive symptoms developed. Physical examination on admission revealed a febrile male in congestive heart failure; the prostate gland was flat and benign. Laboratory data demonstrated a hematocrit of 35 , a hemoglobin of $11 \mathrm{Gm}$. per $100 \mathrm{ml}$, and a white blood count of 17,100 cells per cubic millimeter. The blood glucose was $476 \mathrm{mg}$, the blood urea nitrogen was $49 \mathrm{mg}$., and the serum creatinine was $1.4 \mathrm{mg}$. per 100 $\mathrm{ml}$. Urinalysis revealed 25 to 30 white blood cells per high-power field with bacteria. No fungal forms were identified. Urine culture showed Proteus mirabilis and enterococcus. Excretory urography demonstrated normal upper tracts (Fig. 1). On the scout film and later films a large, laminated, partly radiodense circular mass was seen in the region of the bladder.

The patient was managed initially with an indwelling Foley catheter and antibiotics and, when medically stable, underwent endoscopy.
This revealed a large epithelialized cavity in the place of the prostate, the roof of which was the vesical trigone containing the ureteral orifices. Two large yellow-brown oval masses were free floating in the bladder. There was no evidence of outflow tract obstruction. Open cystotomy was then performed, and two large

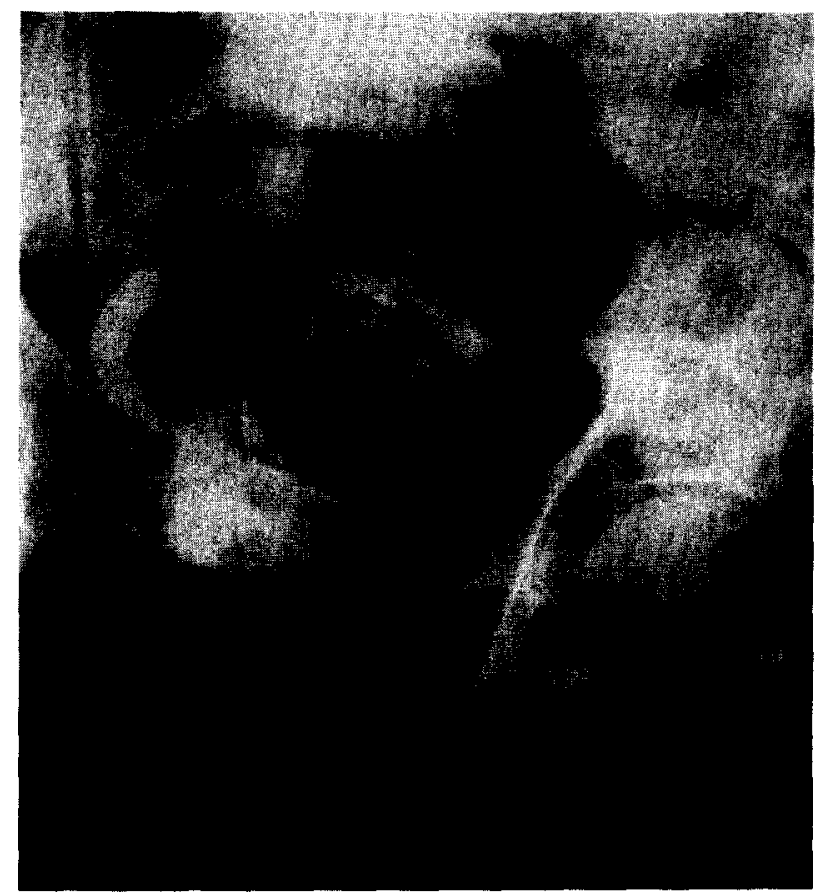

FIGURE 1. Cystographic appearance of fungus ball showing laminated mass. 


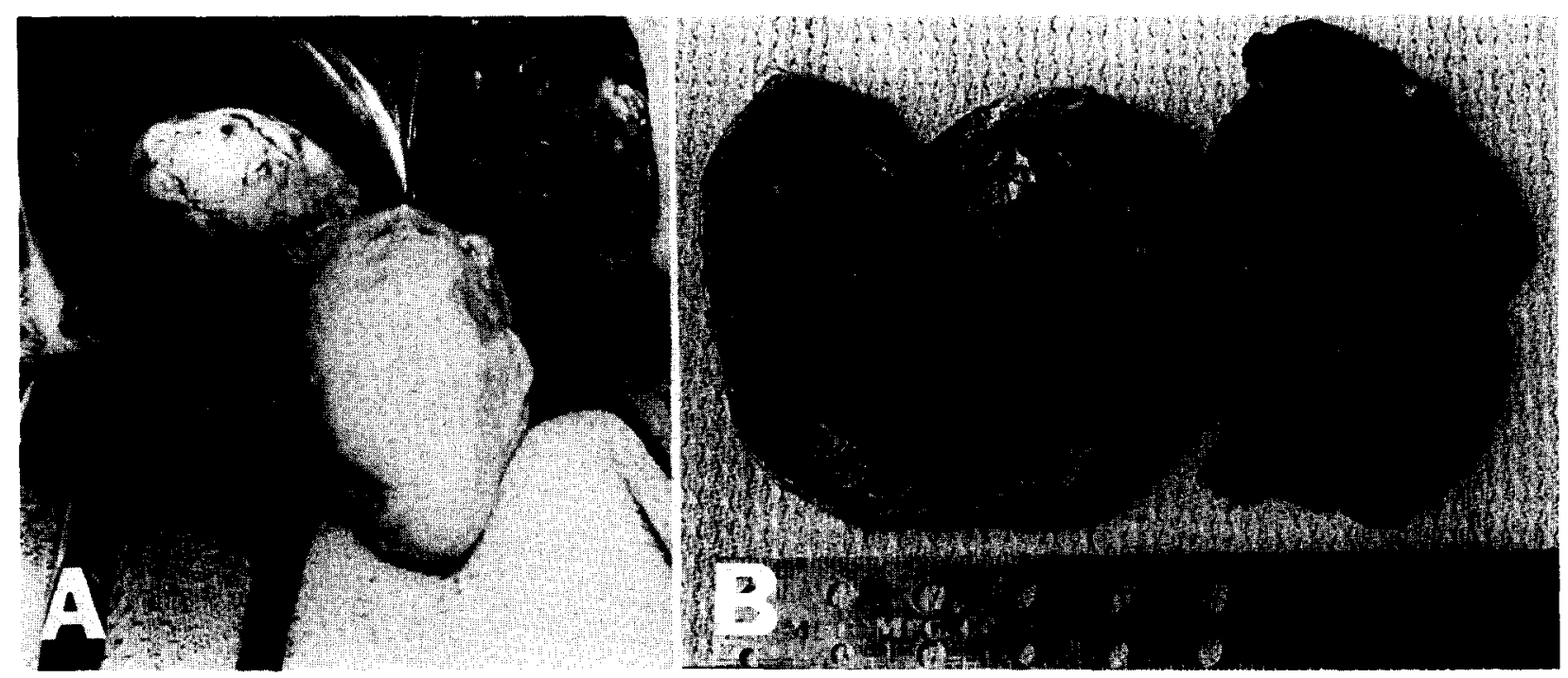

Figure 2. (A) Operative removal of two fungal masses from urinary bladder. (B) Cross section and dimensions of fungal masses.

fungus balls were removed. Culture of these masses revealed Candida albicans, and histologic confirmation of budding yeast was obtained. Two weeks postoperatively after catheter removal the patient voided freely and has remained free of obstructive symptoms (Fig, 2).

\section{Comment}

With the availability and frequent use of potent antibacterial agents, fungal superinfections have become increasingly common especially in diabetic and debilitated patients. Although the urinary tract is susceptible to fungal growth, this colonization is usually clinically inconsequential although tissue invasion may at times occur. ${ }^{1,2}$ Rarely, large colonies of fungi may develop in the bladder and renal pelvis and produce obstructive symptoms. ${ }^{3,4}$

Although the exact pathogenesis of the fungal masses in this case is not entirely clear, we suspect that they are related to the previous prostatic abscess. Internal drainage of this abscess probably introduced a large inoculum of fungal colonies into the bladder, perhaps already in the form of giant fungal masses. The patient's symptoms were due to fungas-ballvalve mechanism with secondary infection.

The radiologic signs of fungal masses in the bladder have been described by Margolin. ${ }^{5}$ These consist of large, laminated, partly radiodense, spheres seen in the bladder region. Although final diagnosis requires histologic and culture confirmation, an accurate preoperative diagnosis can be made on urographic findings alone.

1073 Barton Drive

Ann Arbor, Michigan 48105

(DR. HAROLD)

References

1. Goldman, H. J., Littiman, M. L., Oppenheimer, G. D., and Glickman, S. J.: Monilial cystitis effective treatment with instillations of amphotericin B, J.A.M.A. 174: 359 (1960).

2. Sauer, H. R., and Metzener, U. R. T.: Thrush infection of the urinary bladder: case report, J. Urol. 59: 38 (1948).

3. Warshawsky, A. B., Keiller, D., and Gittes, R. F.: Bilateral renal aspergellosis, ibid. 113: 8 (1975).

4. Chisholm, E. R., and Hutch, J. A.: Fungus ball (Candida albicans) formation in the bladder, ibid. 86: 559 (1961).

5. Margolin, H. N.: Fungus infections of the urinary tract, Semin. Roentgenol. 6: 323 (1971). 